\title{
Extensions of the Signal Richness Preservation Problem in LTI Systems
}

\author{
Borching $\mathrm{Su}$ \\ Department of Electrical Engineering \\ California Institute of Technology \\ Pasadena, California 91125 \\ Email: borching@systems.caltech.edu
}

\author{
P. P. Vaidyanathan \\ Department of Electrical Engineering \\ California Institute of Technology \\ Pasadena, California 91125 \\ Email: ppvnath@systems.caltech.edu
}

\begin{abstract}
The idea of signal richness has recently been studied in considerable details, and the conditions on linear time invariant (LTI) systems to preserve such richness have been established. In this paper, the concept of richness is extended to practically useful classes such as bounded signals. The class of LTI systems studied for the richness preservation problem are generalized to cases including rectangular systems and infinite impulse response (IIR) systems. Structured proofs of the newly found necessary and sufficient conditions are also presented. ${ }^{1}$
\end{abstract}

\section{INTRODUCTION}

Vectorized signals are often considered to be "rich" if they satisfy certain fullness properties appropriate for an application under discussion. In some applications a sequence of $M \times 1$ vectors $\mathbf{x}(n), n \geq 0$ is defined to be rich if the matrix

$$
\left[\begin{array}{llll}
\mathbf{x}(0) & \mathbf{x}(1) & \cdots & \mathbf{x}\left(K_{x}\right)
\end{array}\right]
$$

has rank $M$ for sufficiently large $K_{x}$ [1]. This property is important, for example, when we try to identify an unknown communication channel from output measurements alone using filter bank precoders [2]. Now, signals are sometimes preconditioned by linear time invariant transformations before they are used in such an application [3]. This leads us to explore the conditions under which the linear precoders will preserve richness of the vectorized signals. Let the linear time invariant (LTI) system be characterized by the polynomial matrix

$$
\mathbf{H}(z)=\sum_{k=0}^{N} \mathbf{h}(k) z^{-k}
$$

so that

$$
\mathbf{y}(n)=\sum_{k=0}^{N} \mathbf{h}(k) \mathbf{x}(n-k) .
$$

We say the system $\mathbf{H}(z)$ is richness-preserving (RP) if for any rich input $\mathbf{x}(n)$, the output $\mathbf{y}(n)$ is also rich.

Other types of signal fullness different from but similar to richness described above can be defined depending on the applications. Necessary and sufficient conditions for LTI systems

\footnotetext{
${ }^{1}$ Work supported in parts by the NSF grant CCF-0428326 and the Moore Fellowship of the California Institute of Technology.
}

to preserve similar yet different definitions of fullness can differ very much. In reference [1], another definition of signal fullness, namely, strict richness has also been considered: a sequence of $M \times 1$ vectors $\mathbf{x}(n)$ is said to be strictly $\operatorname{rich}(\mathrm{SR})$ if for any nonnegative integer $n_{0}$, there exists an integer $K_{n_{0}}$ such that the matrix

$$
\left[\begin{array}{llll}
\mathbf{x}\left(n_{0}\right) & \mathbf{x}\left(n_{0}+1\right) & \cdots & \mathbf{x}\left(n_{0}+K_{n_{0}}\right)
\end{array}\right]
$$

has rank $M$. A system that preserves strict richness is called a strict-richness preserving (SRP) system.

The necessary and sufficient conditions for LTI systems to preserve richness and strict richness have been found for the case of $M \times M$ FIR systems [1]. In this paper, we will consider a third definition, namely bounded strict richness, and find the necessary and sufficient conditions for LTI systems to preserve bounded strict richness. We also generalize the richness preservation problem for rectangular systems (i.e., $P \times M$ systems) and infinite impulse response (IIR) systems.

The paper is organized as follows. In Section II, previous results for the richness-preserving problem will be briefly reviewed. In Section III we will define bounded strict richness and present the necessary and sufficient conditions for LTI systems to preserve bounded strict richness. In Sections IV and $\mathrm{V}$, we will reconsider the richness preservation problem and generalize the results for rectangular systems and IIR systems, respectively. The proofs of theorems presented in the Sections IV and V will be given in Section VI. Conclusions and open issues will be described in Section VII.

\section{A. Notations}

Boldfaced lower case letters represent column vectors, and boldfaced upper case letters are reserved for matrices. Superscripts as in $\mathbf{A}^{T}$ and $\mathbf{A}^{\dagger}$ denote the transpose and transposeconjugate operations, respectively, of a matrix or a vector. $[\mathbf{v}]_{i}$ denotes the $i$ th element of vector $\mathbf{v}$, and $\mathbf{e}_{i}$ denotes the $i$ th vector of the standard basis of $\mathbb{C}^{M}$. $[\mathbf{A}]_{i}$ denotes the $i$ th row of matrix $\mathbf{A}$. All the vectors and matrices in this context are complex-valued. 


\section{REVIEW OF RECENT RESUlTS}

In reference [1], conditions on LTI systems to preserve richness have been presented:

Theorem 1: An $N$ th order, $M \times M$ system

$$
\mathbf{H}(z)=\sum_{k=0}^{N} \mathbf{h}(k) z^{-k}
$$

is an RP system if and only if either one of the following conditions is true:

(a) There exist a nonsingular $M \times M$ matrix $\mathbf{A}$ and constants $g_{0}, g_{1}, \cdots, g_{N}$ of which at least one is nonzero such that $\mathbf{h}(k)=g_{k} \mathbf{A}$.

(b) There exist a nonzero row vector $\mathbf{v}^{\dagger}$ and a set of column vectors $\mathbf{a}_{0}, \mathbf{a}_{1}, \cdots, \mathbf{a}_{N}$ such that $\mathbf{h}(k)=\mathbf{a}_{k} \mathbf{v}^{\dagger}$ for any $k$, and $\left[\begin{array}{llll}\mathbf{a}_{0} & \mathbf{a}_{1} & \cdots & \mathbf{a}_{N}\end{array}\right]$ has full rank $M$.

For convenience, we call LTI systems which have the form described in condition (a) Type-A RP systems, and those which have the form in condition (b) Type-B RP systems. As we can observe in the above description, the rank of any nonzero coefficient matrix of a Type-A RP system is always $M$. The rank of any nonzero coefficient matrix of a Type-B RP system is always unity. There are no systems other than Type-A and Type-B RP systems that preserve signal richness.

Conditions on LTI systems to preserve strict richness have also been found [1]:

Theorem 2: An $N$ th order, $M \times M$ system

$$
\mathbf{H}(z)=\sum_{k=0}^{N} \mathbf{h}(k) z^{-k}
$$

is an SRP system if and only if there exists nonnegative integer $n$ and an invertible $M \times M$ matrix $\mathbf{A}$ such that $\mathbf{H}(z)=z^{-n} \mathbf{A}$.

In view of this theorem, we find that an SRP system is also RP (Type-A RP, in fact). In the rest of the this paper, we will extend these existing results in several different directions.

\section{BOUNDED STRICT RICHNESS}

As described in Section II, an SRP system must also be an RP system. For RP systems which do not have the form of an SRP system, strictly rich input sequences can be given to show that the outputs are not strictly rich [1]. In some of these counterexamples, the input signals are unbounded in time. This leads us to think: if we consider only bounded signals, will the conditions to preserve strict richness be different? A sequence of $M \times 1$ vectors $\mathbf{x}(n)$ is said to be bounded strictly-rich (BSR) if $\mathbf{x}(n)$ is strictly rich and there exists a constant $c>0$ such that

$$
\left|[\mathbf{x}(n)]_{i}\right|<c,
$$

for all $n \geq 0$ and $1 \leq i \leq M$. A system that preserves bounded strict richness is called a bounded-strict-richness preserving
(BSRP) system. One of the main results of this paper is the following:

Theorem 3: An $N$ th order, $M \times M$ polynomial matrix

$$
\mathbf{H}(z)=\sum_{k=0}^{N} \mathbf{h}(k) z^{-k}
$$

is a bounded-strict-richness preserving (BSRP) system if and only if it can be expressed as $\mathbf{H}(z)=g(z) \mathbf{A}$, where $\mathbf{A}$ is a nonsingular $M \times M$ matrix and $g(z)$ is an $N$ th order polynomial in $z^{-1}$ whose zeros are all outside the unit circle.

The proof of Theorem 3 requires the following lemmas.

\section{Lemma 1: A BSRP system is also an RP system.}

Proof: We argue that a non-RP system is not a BSRP system. For a non-RP system $\mathbf{H}(z)=\sum_{k=0}^{N} \mathbf{h}(k) z^{-k}$, we can find a rich input $\mathbf{x}(n)$ which has a finite support such that the output is non-rich [1]. Suppose $\mathbf{x}(n)$ is rich and zero for $n \geq L$ for some integer $L$, and the output $\mathbf{y}(n)=\sum_{k=0}^{N} \mathbf{h}(k) \mathbf{x}(n-k)$ is non-rich. We can construct a new input signal

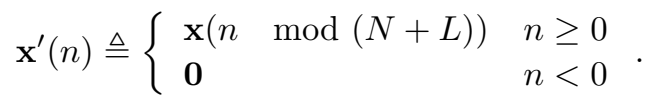

By definition, $\mathbf{x}^{\prime}(n)$ is strictly rich. And since $\mathbf{x}(n)$ has a finite support, it is bounded and hence $\mathbf{x}^{\prime}(n)$ is also bounded. Now using the fact that $\mathbf{x}(n)=\mathbf{0}$ for all $n \geq L$ and $n<0$, we find the output

$$
\begin{aligned}
\mathbf{y}^{\prime}(n) & =\sum_{k=0}^{N} \mathbf{h}(k) \mathbf{x}^{\prime}(n-k) \\
& =\sum_{k=0}^{N} \mathbf{h}(k) \mathbf{x}((n-k) \bmod (N+L)) \\
& =\sum_{k=0}^{N} \mathbf{h}(k) \mathbf{x}([n \bmod (N+L)]-k) \\
& =\mathbf{y}(n \bmod (N+L))
\end{aligned}
$$

is not rich. Hence $\mathbf{y}^{\prime}(n)$ is not bounded strictly-rich though $\mathbf{x}^{\prime}(n)$ is. So $\mathbf{H}(z)$ is not a BSRP system.

Lemma 2: A Type-B RP system is not a BSRP system.

Proof: Suppose $\mathbf{H}(z)=\left(\sum_{k=0}^{N} \mathbf{a}_{k} z^{-k}\right) \mathbf{v}_{1}^{\dagger}$ is a Type-B RP system where $\mathbf{v}_{1}^{\dagger}$ is a nonzero row vector. Without loss of generality, assume $\left\|\mathbf{v}_{1}\right\|=1$. We can find $\mathbf{v}_{2}, \mathbf{v}_{3}, \cdots \mathbf{v}_{M}$ such that $\left\|\mathbf{v}_{k}\right\|=1$ and $\mathbf{v}_{i}^{T} \mathbf{v}_{j}=0, \forall i \neq j$. Let $\mathbf{w}_{1}=\mathbf{v}_{1}$ and $\mathbf{w}_{k}=\mathbf{v}_{1}+\mathbf{v}_{k}$ for $2 \leq k \leq M$. Then we have $\mathbf{v}_{1}^{T} \mathbf{w}_{k}=1, \forall k$. Let the input $\mathbf{x}(n)=\mathbf{w}_{(n \bmod M)+1}$. Clearly it is bounded 
and strictly rich. However, for all $n \geq N$, we have

$$
\mathbf{y}(n)=\sum_{k=0}^{N} \mathbf{a}_{k} \mathbf{v}_{1}^{T} \mathbf{x}(n-k)=\sum_{k=0}^{N} \mathbf{a}_{k},
$$

independent from $n$. This implies $\mathbf{y}(n)$ is not strictly rich. So a Type-B RP system is not a BSRP system.

Lemma 3: An $N$ th order Type-A RP system $\mathbf{H}(z)=g(z) \mathbf{A}$ is a BSRP system if and only if all zeros of $g(z)$ are outside the unit circle.

Proof: Suppose $\mathbf{H}(z)=g(z) \mathbf{A}$ is a Type-A RP system. Here $g(z)$ is an $N$ th order polynomial in $z^{-1}$ and $\mathbf{A}$ is an $M \times M$ invertible matrix. Suppose $g(z)$ has a zero on or inside the unit circle, viz., $g(\alpha)=0$ for some $\alpha$ satisfying $|\alpha| \leq 1$. Let the input $\mathbf{x}(n)=\alpha^{n} \mathbf{A}^{-1} \mathbf{e}_{(n \bmod M)+1}$. By definition, $\mathbf{x}(n)$ is bounded and strictly rich. Then for all $n \geq N$, we have

$$
\begin{aligned}
\sum_{i=1}^{M}[\mathbf{y}(n)]_{i} & =\sum_{i=1}^{M} \mathbf{e}_{i}^{T}\left[\sum_{k=0}^{N} g_{k} \mathbf{A} \mathbf{x}(n-k)\right] \\
& \left.=\sum_{k=0}^{N} g_{k} \alpha^{n-k}\left[\sum_{i=1}^{M} \mathbf{e}_{i}^{T} \mathbf{e}_{((n-k)} \bmod M\right)+1\right] \\
& =\alpha^{n} g(\alpha)=0 .
\end{aligned}
$$

This means the row vector $\left[\begin{array}{llll}1 & 1 & \cdots & 1\end{array}\right]$ is an annihilator of $\mathbf{y}(n)$ for all $n \geq N$. Therefore $\mathbf{y}(n)$ is not strictly rich. So $\mathbf{H}(z)=g(z) \mathbf{A}$ is not a BSRP system if $g(\alpha)=0$ for some $|\alpha| \leq 1$.

On the other hand, if all zeros of $g(z)$ are outside the unit circle, viz., $g(\alpha)=0$ implies $|\alpha|>1$, we will show that $\mathbf{H}(z)=g(z) \mathbf{A}$ is a BSRP system. Assume the contrary. Then there exists a bounded strictly-rich input signal $\mathbf{x}(n)$ such that the output signal $\mathbf{y}(n)=\sum_{k=0}^{N} g_{k} \mathbf{A x}(n-k)$ is not. Without loss of generality, assume $g_{0} \neq 0$ since delays do not affect the property of BSRP. Observe that $\mathbf{H}(z)$ is FIR and hence $\mathbf{y}(n)$ is bounded since $\mathbf{x}(n)$ is. So $\mathbf{y}(n)$ is not strictly rich. Then there exists a nonzero row vector $\mathbf{v}^{\dagger}$ and a nonnegative integer $n_{0}$ such that $\mathbf{v}^{\dagger} \mathbf{y}(n)=0$ for all $n \geq n_{0}$. So we have

$$
\begin{aligned}
0=\mathbf{v}^{\dagger} \mathbf{y}(n) & =\sum_{k=0}^{N} g_{k} \mathbf{v}^{\dagger} \mathbf{A} \mathbf{x}(n-k) \\
& =\sum_{k=0}^{N} g_{k} x_{n-k} \\
& =g_{0} x_{n}+\sum_{k=1}^{N} g_{k} x_{n-k}
\end{aligned}
$$

where we define $x_{n} \triangleq \mathbf{v}^{\dagger} \mathbf{A} \mathbf{x}(n)$. This implies

$$
x_{n}=-\frac{1}{g_{0}}\left(g_{1} x_{n-1}+\cdots+g_{N} x_{n-N}\right)
$$

for all $n \geq n_{0}$. If $\alpha_{k}$ is a zero of $g(z)=\sum_{k=0}^{N} g_{k} z^{-k}$, then clearly $x_{n}=\alpha_{k}^{n}$ is a solution of (1). If $g(z)$ has multiple zeros, then (1) also has solutions of the form $n^{i} \alpha_{k}^{n}$ for some positive integer $i$. So $x_{n}$ is a linear combination of terms of the form $n^{i} \alpha_{k}^{n}$ and can be expressed as

$$
x_{n}=\sum_{k=1}^{N_{1}} \sum_{i=1}^{r_{k}} c_{k, i} n^{i-1} \alpha_{k}^{n}, \quad n \geq n_{0}
$$

where $N_{1}$ is the number of distinct zeros of $g(z)$ and $r_{k}$ is the multiplicity of the zero $\alpha_{k}$. Since $\mathbf{x}(n)$ is SRP and $\mathbf{v}^{\dagger} \mathbf{A}$ is a nonzero row vector, $x_{n}$ must be nonzero for infinitely many $n$. This implies at least one of the coefficients $c_{k, i}$ must be nonzero. So $x_{n}$ is necessarily unbounded as $n$ goes to infinity. This contradicts the BSR assumption on $\mathbf{x}(n)$.

Proof of Theorem 3: From Lemmas 1, 2, and 3, the proof of Theorem 3 is immediately complete.

\section{ReCtangular Systems}

In Section II only $M \times M$ systems are considered for the richness-preservation problem. In this section, we will generalize these results to $P \times M$ systems where $P$ and $M$ might be different.

Theorem 4: An $N$ th order, $P \times M$ system

$$
\mathbf{H}(z)=\sum_{k=0}^{N} \mathbf{h}(k) z^{-k}
$$

is an RP system if and only if either one of the following conditions is true:

(a) There exist a right invertible $P \times M$ matrix $\mathbf{A}$ and constants $g_{0}, g_{1}, \cdots, g_{N}$ of which at least one is nonzero such that $\mathbf{h}(k)=g_{k} \mathbf{A}$.

(b) There exist a nonzero $1 \times M$ vector $\mathbf{v}^{\dagger}$ and a set of $P \times 1$ vectors $\mathbf{a}_{0}, \mathbf{a}_{1}, \cdots, \mathbf{a}_{N}$ such that $\mathbf{h}(k)=\mathbf{a}_{k} \mathbf{v}^{\dagger}$ for any $k$, and $\left[\begin{array}{cccc}\mathbf{a}_{0} & \mathbf{a}_{1} & \cdots & \mathbf{a}_{N}\end{array}\right]$ has rank $P$.

A $P \times M$ matrix $\mathbf{A}$ is said to be right invertible if there exists an $M \times P$ matrix $\mathbf{B}$ such that $\mathbf{A B}=\mathbf{I}_{P}$. It is equivalent to saying that $\operatorname{rank}(\mathbf{A})=P$ or to saying that the $P$ rows of the matrix $\mathbf{A}$ are linearly independent. Note that right invertibility implies $P \leq M$. Thus, according to Theorem 4, there are no Type-A RP systems whose number of outputs is greater than the number of inputs. This fits out intuition that a lower-rank input cannot turn into a higher-rank output.

This is, however, not the case for Type-B RP systems. According to the description in the theorem, even when $P>M$, there still exist systems that satisfy condition (b). However, it is necessary that the order of the system $N$ be greater than or equal to the size of the outputs $P$.

Theorem 5: An $N$ th order, $P \times M$ system

$$
\mathbf{H}(z)=\sum_{k=0}^{N} \mathbf{h}(k) z^{-k}
$$


is a strict-richness preserving (SRP) system if and only if there exist a nonnegative integer $n$ and a right invertible $P \times M$ matrix $\mathbf{A}$ such that $\mathbf{H}(z)=z^{-n} \mathbf{A}$.

The proofs of Theorems 4 and 5 will be presented in Section VI.

\section{Infinite Impulse Response Systems}

In Theorem 1 reviewed in Section II, only FIR systems were considered. In this section we generalize the results for IIR systems.

Theorem 6: A $P \times M$ system

$$
\mathbf{H}(z)=\sum_{k=0}^{\infty} \mathbf{h}(k) z^{-k}
$$

is an RP system if and only if either one of the following conditions is true:

(a) There exist a right invertible $P \times M$ matrix $\mathbf{A}$ and a sequence of scalars $\left\{g_{k}\right\}_{k=0}^{\infty}$ of which at least one scalar is nonzero such that $\mathbf{h}(k)=g_{k} \mathbf{A}$.

(b) There exist a nonzero $1 \times M$ vector $\mathbf{v}^{\dagger}$ and a $P \times 1$ rich sequence $\mathbf{a}(n)$ such that $\mathbf{h}(k)=\mathbf{a}(k) \mathbf{v}^{\dagger}$ for any $k$.

In view of the theorem, the conditions for IIR systems to preserve richness look exactly the same as those for FIR systems. However, it takes extra effort to prove this (See Lemmas 5 and 8 in Section VI). This will be done in Section VI.

Since conditions (a) and (b) described in Theorem 1 are special cases of those in Theorems 4 and 6 , we can extend the definitions of Type-A RP and Type-B RP systems to those which have the forms in conditions (a) and (b) in Theorem 6, respectively.

\section{Proofs of Theorems 4, 5, AND 6}

Theorems 4, 5, and 6 can be proved by the following lemmas. Many of them are generalized from the lemmas in [1] which we have used to prove the case for FIR $M \times M$ systems.

Lemma 4: A $P \times M$ system $\mathbf{H}(z)$ is $\mathrm{RP}$ if and only if $z^{-k} \mathbf{A H}(z) \mathbf{B}$ is $\mathrm{RP}$, where $k$ is any nonnegative integer, $\mathbf{A}$ is a nonsingular $P \times P$ matrix, and $\mathbf{B}$ is a nonsingular $M \times M$ matrix.

Proof: This lemma becomes obvious when we recognize that invertible row operations and delays on input and output signals do not alter the property of richness.

Lemma 5: Suppose a $P \times M$ system $\mathbf{H}(z)=\sum_{k=0}^{N} \mathbf{h}(k) z^{-k}$ is RP, where $N$ is possibly infinite. Then there exist $P \times P$ diagonal matrices $\mathbf{D}_{k}$ and a $P \times M$ constant matrix $\mathbf{A}$ (not necessarily right invertible), each row of which is nonzero, such that $\mathbf{h}(k)=\mathbf{D}_{k} \mathbf{A}$ for all $k$.

Proof: If $N$ is a finite integer, the proof can be easily obtained by extending the proof of Lemma 6 in [1]. If $N$ is infinity, we need to show that for any $i, 1 \leq i \leq P$, the $i$ th rows of all coefficient matrices $\mathbf{h}(k)$ are proportional to the same nonzero row vector which is the $i$ th row of A. Focusing on the $i$ th rows of $\mathbf{h}(k)$ 's, we use

$$
\mathbf{b}_{k}^{T}=\left[\begin{array}{llll}
b_{1 k} & b_{2 k} & \cdots & b_{M k}
\end{array}\right]
$$

to denote the $i$ th row of $\mathbf{h}(k)$ for simplicity. Using Lemma 4 and setting the $M \times M$ matrix $\mathbf{B}$ to appropriate values, we can assume $\mathbf{b}_{0}^{T}=\mathbf{e}_{1}^{T}$ without loss of generality. Now we want to argue that $\mathbf{b}_{k}^{T}$ is proportional to $\mathbf{e}_{1}^{T}$ for all $k \geq 1$. That is, $b_{j k}=0$ for all $k>0$ and $j>1$. Assume the contrary and let $l$ be the smallest integer such that $\mathbf{b}_{l}^{T}$ violates this property (i.e., not proportional to $\mathbf{e}_{1}^{T}$ ). So $b_{j l} \neq 0$ for some $j>1$ and $b_{j k}=0$ for all $j$ and $k$ satisfying $0 \leq k<l$ and $2 \leq j \leq M$.

Now we construct an input $\mathbf{x}(n)$ as follows:

$$
\begin{aligned}
\mathbf{x}(0) & =\mathbf{e}_{j} \\
\mathbf{x}(k) & =\mathbf{0} \text { for } 1 \leq k \leq l-1 \\
\mathbf{x}(l) & =-b_{j l} \mathbf{e}_{1} \\
\mathbf{x}(k) & =\mathbf{e}_{k-l+1}+a_{k} \mathbf{e}_{1} \text { for all } l+1 \leq k \leq l+M-1 \\
\mathbf{x}(k) & =a_{k} \mathbf{e}_{1} \text { for } k \geq l+M
\end{aligned}
$$

where $a_{k}, k \geq l+1$ are coefficients to be determined later. Note that $\mathbf{x}(n)$ is rich regardless of the values of $a_{k}$ 's. Now the $i$ th entries of the output signal can be found as follows:

$$
\begin{aligned}
{[\mathbf{y}(0)]_{i} } & =\mathbf{e}_{1}^{T} \mathbf{e}_{j}=0 \\
{[\mathbf{y}(k)]_{i} } & =\mathbf{e}_{1}^{T} \mathbf{0}+\mathbf{0}^{T} \mathbf{0}+\cdots+\mathbf{0}^{T} \mathbf{0}+\mathbf{0}^{T} \mathbf{e}_{j} \\
& =0 \text { for } 1 \leq k \leq l-1 \\
{[\mathbf{y}(l)]_{i} } & =\mathbf{b}_{0}^{T} \mathbf{x}(l)+\cdots+\mathbf{b}_{l}^{T} \mathbf{x}(0) \\
& =\mathbf{e}_{1}^{T}\left(-b_{j l} \mathbf{e}_{1}\right)+b_{j l}=0 \\
{[\mathbf{y}(k)]_{i} } & =\mathbf{b}_{0}^{T} \mathbf{x}(k)+\sum_{m=1}^{k-1} \mathbf{b}_{m}^{T} \mathbf{x}(k-m) \\
& =\mathbf{e}_{1}^{T}\left(a_{k} \mathbf{e}_{1}\right)+\sum_{m=1}^{k-1} \mathbf{b}_{m}^{T} \mathbf{x}(k-m) \\
& =a_{k}+\sum_{m=1}^{k-1} \mathbf{b}_{m}^{T} \mathbf{x}(k-m) \\
& =0 \text { for all } k \geq l+1
\end{aligned}
$$

if we choose $a_{k}=-\sum_{m=1}^{k-1} \mathbf{b}_{m}^{T} \mathbf{x}(k-m)$ recursively, for all $k \geq l+1$. Note that $\mathbf{x}(k-m), 1 \leq m \leq k-1$, depend on the values of $a_{n}, n \leq k-1$.

So $\mathbf{y}(n)$ is not rich though $\mathbf{x}(n)$ is rich. This contradicts the assumption that $\mathbf{H}(z)$ is RP. Thus the $i$ th rows of all 
coefficient matrices $\mathbf{h}(k)$ should be proportional to the same nonzero $1 \times M$ vector, say $\mathbf{a}_{i}^{T}$. Suppose $[\mathbf{h}(k)]_{i}=d_{k i} \mathbf{a}_{i}^{T}$. Now simply assign $\mathbf{D}_{k}=\operatorname{diag}\left(d_{k 1}, \cdots, d_{k P}\right)$ and $\mathbf{A}=\left[\begin{array}{llll}\mathbf{a}_{1} & \mathbf{a}_{2} & \cdots & \mathbf{a}_{P}\end{array}\right]^{T}$, then the proof of the lemma is complete.

Lemma 6: For a $P \times M$ RP system $\mathbf{H}(z)=\sum_{k=0}^{\infty} \mathbf{h}(k) z^{-k}$, the ranks of all nonzero coefficient matrices must be the same. We call this value the coefficient rank of an RP system.

Lemma 7: The coefficient rank of a $P \times M$ RP system $\mathbf{H}(z)=\sum_{k=0}^{\infty} \mathbf{h}(k) z^{-k}$ can only be either unity of $P$.

Proofs of Lemmas 6 and 7 can be obtained by slightly modifying the proofs of Lemmas 9, 10, and 11 in [1].

Lemma 8: Consider a $P \times M \mathrm{RP}$ system $\mathbf{H}(z)=$ $\sum_{k=0}^{\infty} \mathbf{h}(k) z^{-k}$. If the coefficient rank of $\mathbf{H}(z)$ is $P$, it must have the form of a Type-A RP system. If the coefficient rank of $\mathbf{H}(z)$ is unity, it must have the form of a Type-B RP system. Proof: If the coefficient rank of $\mathbf{H}(z)$ is $P$, by Lemma 5 $\mathbf{h}(k)=\mathbf{D}_{k} \mathbf{A}$ for all $k$, where $\mathbf{D}_{k}$ are diagonal matrices. Since $\operatorname{rank}(\mathbf{h}(k))=P$ for all nonzero $\mathbf{h}(k)$, we have $\operatorname{rank}(\mathbf{A})$ $=P$. Suppose $\mathbf{a}_{1}^{T}, \mathbf{a}_{2}^{T}, \ldots, \mathbf{a}_{P}^{T}$ are rows of $\mathbf{A}$. They are linearly independent. For convenience, let $\mathbf{d}_{k}$ be a $P \times 1$ vector whose entries are diagonal entries of $\mathbf{D}_{k}$. We argue that all vectors $\mathbf{d}_{k}=\left[\begin{array}{llll}d_{k 1} & d_{k 2} & \cdots & d_{k P}\end{array}\right]^{T}$ are in the same direction. Assume the contrary and suppose $\mathbf{d}_{k}$ and $\mathbf{d}_{l}$ are not proportional to each other: $\exists i, j$ such that $d_{k i} d_{l j} \neq d_{l i} d_{k j}$. Recall that $[\mathbf{h}(k)]_{i}$ denotes the $i$ th row of matrix $\mathbf{h}(k)$. Now we have $[\mathbf{h}(k)]_{i}=d_{k i} \mathbf{a}_{i}^{T},[\mathbf{h}(k)]_{j}=d_{k j} \mathbf{a}_{j}^{T},[\mathbf{h}(l)]_{i}=d_{l i} \mathbf{a}_{i}^{T}$, and $[\mathbf{h}(l)]_{j}=d_{l j} \mathbf{a}_{j}^{T}$. By Lemma 4 we know that $\mathbf{H}^{\prime}(z) \triangleq \mathbf{K H}(z)$ is also an RP system where $\mathbf{K}$ is chosen as an invertible matrix which adds the $i$ th row of $\mathbf{H}(z)$ into the $j$ th row of it. This implies $\left[\mathbf{h}^{\prime}(k)\right]_{j}=d_{k i} \mathbf{a}_{i}^{T}+d_{k j} \mathbf{a}_{j}^{T}$ and $\left[\mathbf{h}^{\prime}(l)\right]_{j}=$ $d_{l i} \mathbf{a}_{i}^{T}+d_{l j} \mathbf{a}_{j}^{T}$. They are in different directions since $\mathbf{a}_{i}$ and $\mathbf{a}_{j}$ are linearly independent and $d_{k i} d_{l j} \neq d_{l i} d_{k j}$. This makes the RP system $\mathbf{H}^{\prime}(z)$ violate Lemma 5. So all $\mathbf{d}_{k}$ must be in the same direction and hence $\mathbf{h}(k)=\mathbf{D}_{k} \mathbf{A}$ are all proportional to the same right invertible matrix. So $\mathbf{H}(z)$ must have the form of a Type-A RP system.

If the coefficient rank of $\mathbf{H}(z)$ is unity, we can conclude $\mathbf{H}(z)$ must have the form of a Type-B RP system by slightly modifying the proof presented in Section VI-F in [1].

Lemma 9: A $P \times M$ system with the form $\mathbf{H}(z)=$ $\sum_{k=0}^{\infty} g_{k} \mathbf{A} z^{-k}$ is RP if $\operatorname{rank}(\mathbf{A})=P$ and some $g_{k}$ is nonzero.

Lemma 10: A $P \times M$ system with the form $\mathbf{H}(z)=\sum_{k=0}^{\infty} \mathbf{a}(k) \mathbf{v}^{\dagger} z^{-k}$ is $\mathrm{RP}$ if $\mathbf{a}(n)$ is rich and $\mathbf{v}^{\dagger}$ is nonzero.
Proofs of Lemmas 9 and 10 can be obtained by slightly modifying the proofs presented in Section VI-B in [1].

Lemma 11: An FIR $P \times M$ SRP system is also RP.

Lemma 12: An FIR $P \times M$ Type A RP system is SRP if and only if it is a constant right invertible matrix with a possible delay.

Lemma 13: An FIR $P \times M$ Type-B RP matrices $\mathbf{B}(z)$ do not preserve strict richness.

The proofs of Lemmas 9, 10, and 11 can be obtained by slightly modifying the proofs of Lemmas 5, 12, and 13 in [1].

Finally, Theorems 4 and 6 can be proved by using Lemmas 6, 7, and 8 (necessary conditions) and Lemmas 9 and 10 (sufficient conditions). The proof of Theorem 5 can be obtained from Lemmas 11, 12, and 13.

\section{CONCluding REmarks AND Open Issues}

In this paper we introduced the idea bounded strictlyrich signals. The conditions for FIR LTI systems to preserve this property have been found. It is worthy to note that a minor change in the definition of strict richness constitutes a significant difference on the conditions to preserve such richness.

We also generalized the richness-preservation problem for rectangular systems and IIR systems. It remains to investigate conditions on infinite impulse response (IIR) systems that preserve strict richness and bounded strict richness.

In the literature on adaptive filtering [4] and control theory [5], a class of signals called the persistently exciting class is often defined. The connection between such signals and the "rich" signals defined herein is discussed in [1]. It will be interesting to extend the results of this paper for the case of persistently exciting signals.

\section{REFERENCES}

[1] Su, B. and Vaidyanathan, P. P., "Theoretical Issues on LTI Systems Which Preserver Signal Richness" IEEE Trans. Signal Processing, in press.

[2] Scaglione, A., Giannakis, G. B., and Barbarossa, S. "Redundant filter bank precoders and equalizers Part II: Synchronization and direct equalization”, IEEE Trans. Signal Processing, vol. 47, pp. 2007-2002, July 1999.

[3] Phoong, S.-M. and Chang, K.-Y., "Antipodal paraunitary matrices and their application to OFDM systems", IEEE Trans. Signal Processing, vol. 53, pp. 1374-1386, April 2005.

[4] Sayed, A. H., Fundamentals of Adaptive Filtering, John Wiley \& Sons, Inc., Hoboken, NJ, 2003.

[5] Sethares, W. A. et al., "Parameter drift in LMS adaptive filters," IEEE Trans. Acoustic., Speech, Signal Processing, vol. ASSP-34, pp. 868-879, August 1986. 